\title{
The European Diploma of Paediatric Radiology: the officers' perspective
}

\author{
Maria Raissaki $^{1}$ (D) Samuel Stafrace ${ }^{2} \cdot$ Jean-François Chateil ${ }^{3} \cdot$ Rutger A. J. Nievelstein $^{4}$
}

Received: 27 October 2021 / Revised: 27 October 2021 / Accepted: 16 November 2021 / Published online: 23 January 2022

(c) The Author(s), under exclusive licence to Springer-Verlag GmbH Germany, part of Springer Nature 2021

\section{Dear Editors,}

Over the last decade, the European Society of Paediatric Radiology (ESPR) has put great effort into enhancing research and education. On Oct. 12, 2021, the first successful paediatric radiologists were officially awarded the European Diploma in Paediatric Radiology (EDiPR) organised by ESPR. This took place during an emotional inaugural event at the end of the ceremonial session at the International Paediatric Radiology 2021 Conference in Rome, which was well-attended and livestreamed worldwide. The ESPR Education Committee officers' perspectives regarding the team efforts of candidates and organisers are shared herein.

The idea of establishing a structured educational program, including a diploma in paediatric radiology, was launched by Jean-François Chateil and members of the ESPR board about 10 years ago. The plans were fueled by the success of the European Society of Neuroradiology (ESNR) and Andrea Rossi. On behalf of the ESPR board, Chateil, Rick van Rijn, Rutger Nievelstein and Samuel Stafrace (as the current Junior ESPR [JESPeR] representative), in collaboration with the members of the board, updated the paediatric radiology chapters of the ESPR training curriculum and

Maria Raissaki

mraissaki@yahoo.gr

1 Department of Radiology, University Hospital of Heraklion, Medical School, University of Crete, Stavrakia Voutes, 71110 Heraklion, Greece

2 Department of Medical Imaging, Sidra Medicine, Doha, Qatar

3 CHU de Bordeaux, Université de Bordeaux, Bordeaux, France

4 Division Imaging \& Oncology, Department of Radiology \& Nuclear Medicine, University Medical Center Utrecht, and Princess Máxima Center for Pediatric Oncology, Utrecht, The Netherlands defined relevant components of level I, II and III radiology knowledge, skills and competencies in Europe. These training curricula have been revised several times by the current ESPR Education Committee, most recently in March 2020, and have been amended and approved by the ESPR board [1].

The ESPR Education Committee was formalised in 2015. Its first chair, Nievelstein, undertook the responsibility of establishing a group of paediatric radiologists and planning actions that would lead to a European Diploma in Paediatric Radiology. The group included (in alphabetical order): Thomas Angell Augdal (Norway), Ignasio Barber (Spain), Pablo Caro Dominguez (Spain, JESPeR representative), Anne Patterson (United Kingdom), Maria Raissaki (Greece), Aurelio Secinaro (Italy), Susan Shelmerdine (United Kingdom), Samuel Stafrace (Qatar) and Eilish Twomey (Ireland). Following discussion, the final structure of the diploma was formulated and announced; the diploma was named the European Diploma in Paediatric Radiology (EDiPR) [2].

In 2018, Raissaki took over as chair of the ESPR Education Committee. Changes in committee members included the addition of Julian Jurgens (Germany, JESPeR representative), Chateil (France), William Ramsden (United Kingdom), Andrea Rossi (Italy), and Michael Paddock (United Kingdom) with Dominguez and Augdal stepping down. The committee discussed the logistical organisation of the diploma while a separate examination subcommittee (from which the junior members of the committee were excluded) discussed and specifically collated the test material.

Since 2017, the ESPR has organised annual European Course Paediatric Radiology (ECPR) courses, with a local organising committee in charge of the program. A written exam including questions on central paediatric issues and harmonised with the European Society of Radiology (ESR) training curriculum, has followed each course. Furthermore, to ensure coverage of the complete ESR training curriculum, learning objectives have been provided for the speakers' guidance and convenience. Importantly, the Education 

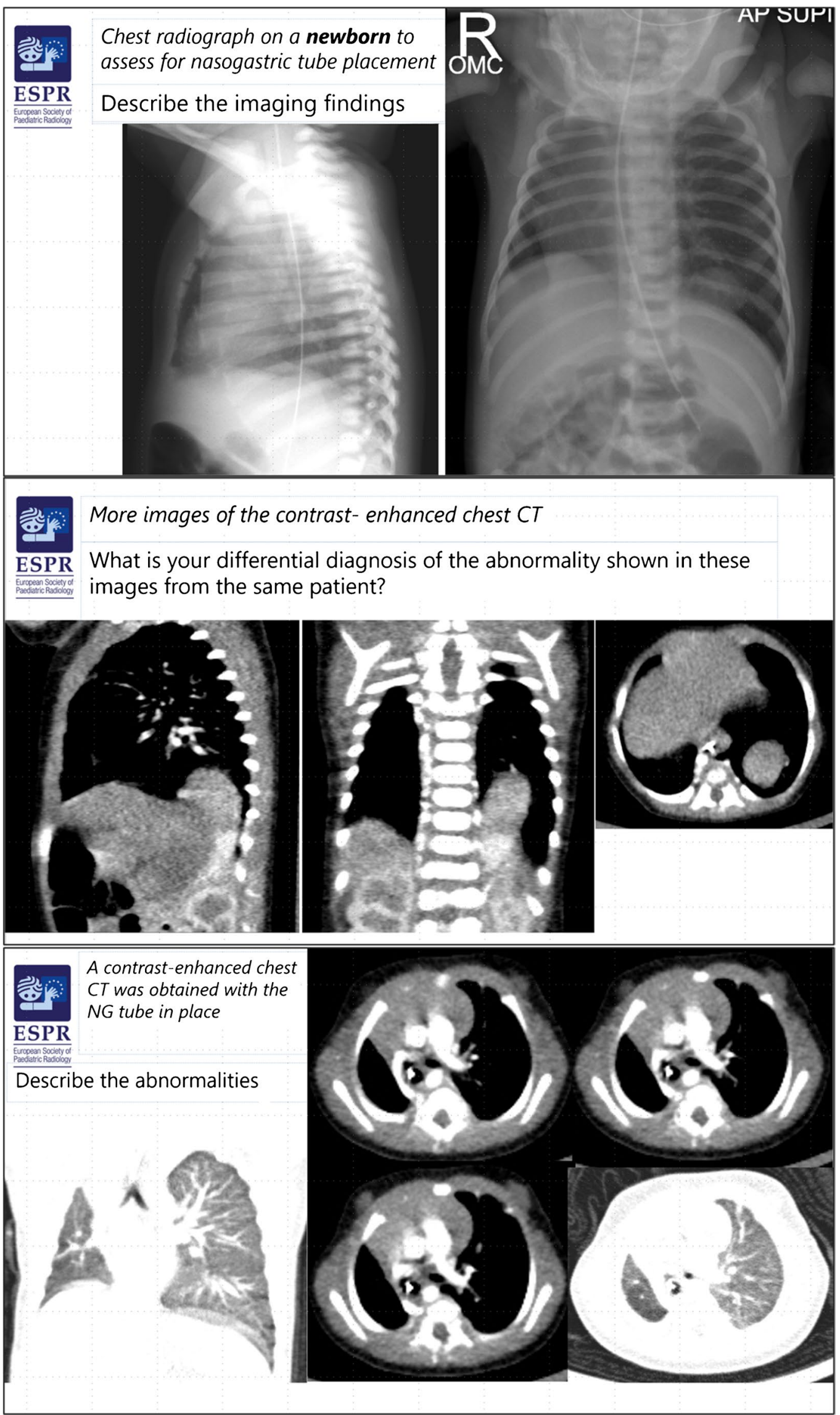
4Fig. 1 An example of a case used for the final diploma oral examination. Identification of key findings such as small vessel calibre and reduced lung volume on the right, mentioning right lung hypoplasia and left Bochdalek or diaphragmatic hernia were important for success

Committee has published a document for ESPR members in good standing interested in organising future European Course Paediatric Radiology courses [3].

Speakers invited to the European Course Paediatric Radiology courses were asked to prepare their lectures and forward their slides well in advance of each course to make the material available to participants. The lectures covered the curriculum-based goals and objectives, consolidating the knowledge required to pass the (multiple choice question) exam. Speakers were also asked to provide 3-6 multiple choice questions and one longer case for the course exam and final oral European Diploma in Paediatric Radiology examination, respectively, by using a PowerPoint template set by the committee.

All questions and cases provided by the speakers were assembled by the ESPR office and reviewed by senior members of the Education Committee. Criteria for inclusion in the examination were clarity of the stem question, adequate justification of answer/s, scientific validity and the possibility of being a discriminating (not too easy) question.

Cases for the oral examination were selected to cover all aspects of paediatric radiology, including neuro/neck, chest/ cardio, abdomen and the musculoskeletal system. Each case consisted of two to six slides, with a short history, images and dedicated questions (Fig. 1). Correct answers were marked with 10 points for each case.

Pass marks of written modular exams were predefined as $60 \%$ of the average of the marks of the three best candidates. On the first sitting (European Course Paediatric Radiology 2017, Utrecht, musculoskeletal test), 61 candidates took the test with a success rate of $84 \%$. On the second sitting (European Course Paediatric Radiology 2018, Dublin, neck chest and heart test), 103 candidates took the test, with a success rate of $85.4 \%$, while on the third sitting (European Course Paediatric Radiology 2019, Utrecht, abdomen), 110 candidates took the test with a success rate of $98 \%$. Following the 2021 Barcelona online musculoskeletal multiple choice question test and the European Course in Paediatric Neuroradiology (ECPNR) tests, 19 candidates passed the three modular tests as well as the neuroradiology test and were thus deemed eligible for the final exam, provided they fulfilled the remaining criteria for application [4]. Ultimately, 17 candidates applied for the diploma exam, all of whom passed the preliminary written multiple choice question test, while 16 passed the viva test.
Due to the coronavirus disease 2019 (COVID-19) pandemic, online examinations had to be considered for the final written and oral tests. The online written multiple choice question test was performed in collaboration with the European Board of Radiology office, which provided the software on which the questions and answers were uploaded. The European Board of Radiology office also provided the proctoring software and technical assistance to proctors and all candidates on the day of the examination. The entire procedure was proctored remotely and ensured continuous monitoring of the screens, faces and environment of candidates during the written test.

Again, in line with the new post-pandemic circumstances, the final oral examination had a hybrid format including a mixture of online and onsite candidates as well as online and onsite examiners.

Two dedicated examiner meetings were held. The agenda for the first, 1 week before the exam, was harmonising the conduct and processes between all the examiners whilst ensuring familiarisation with the examination material. The second examiner meeting occurred on the evening before the final oral examination, with an overview of the setup of the technical equipment and simulation of examination conditions to ensure a smooth performance with the digital platforms. Organisation required four rooms equipped with computers and technical assistance from an onsite technician and the European Board of Radiology office.

The schedule was arranged in such a way that no candidate was examined by an examiner from the same country of origin and that at least one examiner was present on site.

All candidates had to log onto the "Proctor-exam" platform to ensure recording of the exam and availability to review the examination performance retrospectively if this was deemed necessary.

Each candidate was examined by two examiners. The first read the questions, stimulated adequate discussion whilst ensuring that no valuable time was unnecessarily wasted by giving the candidate the opportunity to answer as many cases as possible within the pre-determined time frame. Both examiners scored the candidates on a marking sheet that contained the maximum points per question, prepared and delivered by the examination subcommittee of the Education Committee. Following completion of the 35-min oral test, marks were totalled and a consensus decision reached by the pair of examiners.

Prospective examiners were selected and invited from ESPR officers, Education Committee members and ESPR members based on their expertise with examinations, delivery of previous lectures during European Course Paediatric Radiology courses, representation of the wider geopolitical distribution in the society, and their availability on the chosen day of the examination. Examiners of the inaugural final 
European Diploma in Paediatric Radiology oral examination included (in alphabetical order) Maria Argyropoulou (Greece), Barber (Spain), Marie Cassart (Belgium), van Rijn (the Netherlands), Raissaki (Greece), Karen Rosendahl (Norway), Stafrace (Doha) and Twomey (Ireland). The guarantor was Nievelstein (the Netherlands), who was available to review and score examinations of candidates with equivocal results.

Although there were technical difficulties that delayed the initiation of some sessions, equal unbiased treatment toward all candidates was achieved. The examination was an engaging experience. It was rewarding to appreciate the concentration and dedication of the candidates paired by their specialist competence. It was equally rewarding to enjoy the similarities in recommended management that the candidates suggested on the challenging cases they were presented: The candidate's success ended up being the examiner's triumph.

It was evident that candidates appreciated the opportunity to work hard, improve and consolidate knowledge. They also enjoyed the added benefit of meeting and getting to know each other in person. Getting to know the ESPR officers made them feel embraced by the ESPR family, recommending the continuation of onsite (in addition to online) courses and congresses. The candidates were offered the opportunity to submit feedback and some anonymised quotations include:

"I have to admit that (when all the stress finally disappeared) I realised - it was fun!"

"It is a great honour to be found worthy of this degree! It is my hope and wish to continue to improve and develop professionally in the field of paediatric radiology!"

"It was very stressful to prepare for the exam but a good way to push oneself to study more than one would do otherwise."

"It is not just the positive benefits of a 'tangible' standard across Europe, but the immersion into a network of colleagues and new friends. I am really happy to have knowledge to use and share."

"This experience was an opportunity to review and sediment all the knowledge that was transmitted during the several courses. It came as a personal investment to take some time to challenge myself."

"This lengthy process covered all fields in paediatric radiology, initiated communication with colleagues of different professional and socioeconomical backgrounds, involved high-quality teaching and was a once-in-a-lifetime experience. The transparent, fair examination process demanded a high level for success, ensuring the diploma's prestige."

For those candidates who have failed or are destined to fail future modular or final viva tests, one must be reminded that the knowledge gained during this process is neither measurable nor wasted. Knowledge and experience acquired during attendance and completion of all modules are the truly gained assets of the European Diploma in Paediatric Radiology journey. Accreditation will be achieved with repeated effort, practice, and progressive experience in paediatric radiology.

For those candidates who already are or are destined to become European Diploma in Paediatric Radiology holders, congratulations are in order. Being an accredited ESPR member and having gone through this process comes with a sense of duty to keep up with expectations. Passing a test does not of itself change one's responsibility to ensure a high-level of paediatric radiology practice but does encourage the formulation of a generation of young radiologists who appreciate the challenges in our field and the benefits of continuous education. Attendance at European Course Paediatric Radiology courses and ESPR congresses is a learning process that follows ESPR members for life.

Besides the benefits of professional career development, accreditation with the European Diploma in Paediatric Radiology comes with the potential to apply and participate as test material reviewer in the Education Committee. Further growth of the society is of utmost importance to the welfare of all children. All the dedicated people behind this diploma were once enthusiastic European Course Paediatric Radiology attendees, and in the not-so-distant future, European Diploma in Paediatric Radiology graduates will be expected to fill their shoes.

Acknowledgements Deep appreciation and gratitude are extended to previous and current Education Committee members for their active involvement in this team effort, continuous support, and feedback; to previous and current ESPR board members for financial support, encouragement, and insight; to ECPR speakers for sending test material; to the ESPR and EBR offices, the proctors and to all examiners. Gratitude is extended to the candidates of the ECPR and ECPNR tests who dared to overcome adversities and participated throughout the cycle, ensuring success at every level. Special thanks to Michael Paddock for language editing.

\section{Declarations}

Conflicts of interest None.

\section{References}

1. European Training Curriculum for Radiology. Training Curricula I European Society of Radiology. https://www.myesr.org/ education/training-curricula. Accessed 24 Oct 2021

2. Stafrace S, Nievelstein RJ, Raissaki M, European Society of Paediatric Radiology Education and Professional Development Committee (2018) The European Society of Paediatric 
Radiology launches European Diploma in Paediatric Radiology. Pediatr Radiol 48:1040-1042

3. The European Society of Pediatric Radiology. Events. Want to organise the next congress or course? https://www.espr.org/ app/uploads/ECPR-Conference-Hosting-Application-Form.pdf. Accessed 24 Oct 2021
4. The European Society of Pediatric Radiology. EDiPR. https:// www.espr.org/edipr-terms-and-conditions_08-2021. Accessed 24 Oct 2021

Publisher's note Springer Nature remains neutral with regard to jurisdictional claims in published maps and institutional affiliations. 